\title{
EXPLICIT FORM OF THE HILBERT SYMBOL FOR POLYNOMIAL FORMAL GROUPS
}

\author{
S. VOSTOKOV AND V. VOLKOV
}

\begin{abstract}
Let $K$ be a local field, $c$ a unit in $K$, and $F_{c}(X, Y)=X+Y+c X Y$ a polynomial formal group that gives rise to a formal module $F_{c}(\mathfrak{M})$ on the maximal ideal in the ring of integers of $K$. Assume that $K$ contains the group $\mu_{F_{c}, n}$ of the roots of isogeny $\left[p^{n}\right]_{c}(X)$. The natural Hilbert symbol $(\cdot, \cdot)_{c}: K^{*} \times F_{c}(\mathfrak{M}) \rightarrow \mu_{F_{c}, n}$ is defined over the module $F(\mathfrak{M})$. An explicit formula for $(\cdot, \cdot)_{c}$ is constructed.
\end{abstract}

\section{§1. INTRODUCTION}

The present paper continues the papers [5, 6], which were devoted to Hilbert pairing over the so-called polynomial formal groups. It is easy to show that the groups of the form $F_{c}=X+Y+c X Y$ are the only formal groups defined by polynomials. We call such groups polynomial formal groups and investigate the Hilbert pairing over them. The case where the element $c$ belongs to the ring of Witte vectors, i.e., the case of polynomial Honda groups, was treated in [5]. There, the main results in the unramified case were formulated together with proof sketches. A similar pairing over the universal formal group $F_{x}=X+Y+x X Y$ (where $x$ is an independent variable) was constructed and studied in [6].

Now we consider an arbitrary polynomial formal group $F_{c}=X+Y+c X Y$, where $c$ is a unit in some local ring (finite extension of $\mathbb{Q}_{p}$ ) and find an explicit formula for the Hilbert symbol defined over the formal module of the group $F_{c}$. Similar formulas were constructed previously for the formal Lubin-Tate groups in [9, 10, for Honda groups in [11, 12, 14, and for the relative formal Lubin-Tate groups in [13]. The case considered in the present paper provides the first example of a formula for the Hilber symbol of groups whose ring of endomorphisms embeds in, but is not isomorphic to the ring where the group is defined.

We use the method suggested in 8 to derive the claimed explicit formula.

\section{$\S 2$. Notation}

We denote:

- $p \geq 3$ is a prime number;

- $\zeta$ is a certain fixed primitive root of unity of degree $p^{n}$;

- $K$ is a finite extension of the field $\mathbb{Q}_{p}$ that contains $\zeta$, with the ring of integers $\mathcal{O}_{K}$;

- $c$ is a unit of the local field $K$;

- $T$ is the inertia subfield of $K$, with the ring of integers $\mathcal{O}=\mathcal{O}_{T}$;

- $e$ is the ramification degree of $K / T$;

- $e_{1}=e /(p-1)$ is the relative ramification degree of $K / T\left(\zeta_{1}\right)$;

- $e_{0}=e_{1} / p^{n-1}$;

2010 Mathematics Subject Classification. Primary 11S31; Secondary 14L05.

Key words and phrases. Polynomial formal groups, formal groups, Hilbert symbol, local rings.

Supported by RFBR (grant no. 14-01-00393). 
- $\triangle$ is the Frobenius automorphism in $T / \mathbb{Q}_{p}$;

- $\operatorname{tr}$ is the trace in $T / \mathbb{Q}_{p}$;

- $\pi$ is a prime element of the field $K$;

- $\mathfrak{M}$ is a maximal ideal of $\mathcal{O}_{K}$;

- $\mathfrak{R}$ is a set of Teichmuller representatives of the residue field $\bar{K}$ in the ring $\mathcal{O}_{K}$;

- $F_{c}=X+Y+c X Y$ is a polynomial formal group;

- $F_{c}(\mathfrak{M})$ is the formal $\mathbb{Z}_{p}$-module on $\mathfrak{M}$, determined by the group $F_{c}$;

- $\lambda_{c}(X)$ is the logartihm of the formal group $F_{c}$;

- $K_{\beta}$ is the extension of the field $K$ constructed by adding roots of the equation $\left[p^{n}\right]_{c}(X)=\beta$ for some $\beta \in F_{c}(\mathfrak{M})$;

- $\xi$ is a generating element of the kernel of $\left[p^{n}\right]_{c}(X)$;

- $\mathrm{d}$ is the operator of differentiation by $X$ in $O((X))$.

It is easily seen that

$$
F_{c}(X, Y)=c^{-1}((1+c X)(1+c Y)-1),
$$

whence $\lambda_{c}(X)=c^{-1} \log (1+c X)$ and $\xi=c^{-1}(\zeta-1)$. We denote the completion of the maximal unramified $p$-extension of $T$ by $\widetilde{T}$, and the Frobenius automorphism of the topological Galois group $\operatorname{Gal}(\widetilde{T} / T)$ by $\varphi$ :

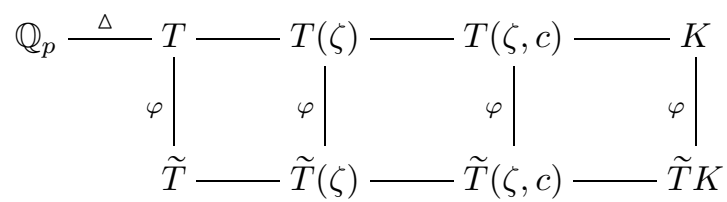

We fix certain series $\underline{c}, \underline{\zeta}$, and $\underline{\xi}=\underline{c}^{-1}(\underline{\zeta}-1)$ in $\mathcal{O}[[X]]$ such that $\underline{c}(\pi)=c, \underline{\zeta}(\pi)=\zeta$, and $\underline{\xi}(\pi)=\xi$.

Consider the group $\mathcal{H}_{\mathfrak{m}}=\mathcal{O}((X))^{*}$ and the formal $\mathbb{Z}_{p}$-module $\mathcal{H}_{\mathrm{c}}=X \mathcal{O}[[X]]$.

The structure of a $\mathbb{Z}_{p}$-module is defined on $\mathcal{H}_{\mathrm{c}}$ by the formal group $F_{\underline{c}}=X+Y+\underline{c} X Y$, which is an analog of the group $F_{c}$, and the series $\lambda_{c}=\underline{c}^{-1} \log (1+\underline{c} \bar{X})$ is an analog of its logarithm.

We introduce an operator $\Delta$ on the Laurent series ring:

$$
\triangle f(x)=\sum a_{i}^{\Delta} X^{p i}, \text { where } f(x)=\sum a_{i} X^{i}, a_{i} \in \mathcal{O} .
$$

Next, we define the series

$$
\begin{aligned}
& \text { - } s_{c}=\left[p^{n}\right]_{c}(\underline{\xi})=\underline{c}^{-1}\left((1+\underline{c} \cdot \underline{\xi})^{p^{n}}-1\right) \text {; } \\
& \text { - } s_{n-1, c}=\left[p^{n-1}\right]_{c}(\underline{\xi}) ; \\
& \text { - } u_{c}=s_{c} / s_{n-1, c} .
\end{aligned}
$$

Note that if we further define $s=\left(\underline{\zeta}^{p^{n}}-1\right)$ and $s_{n-1}=\left(\underline{\zeta}^{p^{n-1}}-1\right)$, where $\underline{\zeta}=1+\underline{c} \cdot \underline{\xi}$, then

$$
u_{c}=s_{c} / s_{n-1, c}=s / s_{n-1}=u,
$$

where $s, s_{n-1}$, and $u$ are the same as in [1, §3].

Consider the following functions $E$ and $\ell$ :

$$
\begin{aligned}
\ell(\alpha) & =\frac{1}{p} \log \left(\frac{\alpha^{p}}{\alpha^{\Delta}}\right), \text { where } \alpha \in \mathcal{H}_{\mathfrak{m}}, \\
E(\beta) & =\exp \left(1+\frac{\triangle}{p}+\frac{\triangle^{2}}{p^{2}}+\ldots\right) \beta, \text { where } \beta \in X \mathcal{O}[[X]]
\end{aligned}
$$

(see [1, §1]). 
The definition given above agrees with that in $[1, \S 1]$, because for $\beta \in X \mathcal{O}[[X]]$ we have

$$
\ell(1+\beta)=\frac{1}{p} \log \left(\frac{(1+\beta)^{p}}{(1+\beta)^{\Delta}}\right)=\log (1+\beta)-\frac{1}{p} \log \left(1+\beta^{\Delta}\right)=\left(1-\frac{\Delta}{p}\right) \log (1+\beta) .
$$

Consider the analogs of these functions for the formal group $F_{\underline{c}}$ :

$$
\begin{aligned}
\ell_{c}(\beta) & =\underline{c}^{-1} \ell(1+\underline{c} \beta), \text { where } \beta \in \mathcal{H}_{\mathrm{c}}, \\
E_{c}(\beta) & =\underline{c}^{-1}(E(\underline{c} \beta)-1), \text { where } \beta \in X \mathcal{O}[[X]] .
\end{aligned}
$$

The corresponding result on the functions $\ell$ and $E$ (see [1, §1, Proposition 1]) implies the following statement.

Proposition 1. The functions $\ell_{c}$ and $E_{c}$ are mutually inverse isomorphims between the additive $\mathbb{Z}_{p}$-module $X \mathcal{O}[[X]]$ and the formal $\mathbb{Z}_{p}$-module $\mathcal{H}_{\mathrm{c}}$.

In what follows, we use the notation $F_{c}$ for the formal group $F_{\underline{c}}$ and $\lambda_{c}$ for its logarithm $\lambda_{\underline{c}}$, since it is always clear whether we are currently dealing with numbers or formal series.

One can easily check the following relations:

$$
\mathrm{d} \alpha^{\Delta}=p X^{-1}(X \mathrm{~d} \alpha)^{\Delta} ; \quad \mathrm{d} \ell(\alpha)=\alpha^{-1} \mathrm{~d} \alpha-X^{-1}\left(X \alpha^{-1} \mathrm{~d} \alpha\right)^{\Delta} .
$$

We also recall the definition of the Hilbert symbol $(\cdot, \cdot)_{c}$ of the formal group $F_{c}$ :

$$
(\alpha, \beta)_{c}=B^{\sigma(\alpha)}-F_{c} B,
$$

where $\alpha \in K^{*}, \beta \in F_{c}(\mathfrak{M}), B$ is the solution of the equation $\left[p^{n}\right]_{c}(B)=\beta$, and $\sigma: K^{*} \rightarrow$ $\operatorname{Gal}\left(K^{\mathrm{ab}} / K\right)$ is the Artin-Frobenius automorphism.

\section{§3. Pairing over the formal $\mathbb{Z}_{p}$-Module $\mathcal{H}_{\mathrm{c}}$}

For $\alpha \in K^{*}$ and $\beta \in F_{c}(\mathfrak{M})$, we introduce the pairing

$$
\begin{aligned}
\langle\cdot, \cdot\rangle_{c}: & \mathcal{H}_{\mathfrak{m}} \times \mathcal{H}_{\mathrm{c}} \rightarrow \mathcal{O} / p^{n}, \\
\alpha, \beta & \mapsto \operatorname{res}_{X} \Phi(\alpha, \beta) / s_{c} \bmod p^{n},
\end{aligned}
$$

where $\Phi(\alpha, \beta)=\ell_{c}(\beta) \alpha^{-1} \mathrm{~d} \alpha-\ell(\alpha) \underline{c}^{-1} \mathrm{~d} \frac{\Delta}{p} \underline{c} \lambda_{c}(\beta)$.

Proposition 2. The pairing $\langle\cdot, \cdot\rangle_{c}$ is $\mathbb{Z}_{p}$-linear, i.e.,

$$
\begin{aligned}
\left\langle\alpha_{1} \alpha_{2}, \beta\right\rangle_{c} & =\left\langle\alpha_{1}, \beta\right\rangle_{c}+\left\langle\alpha_{2}, \beta\right\rangle_{c} ; & & \left\langle\alpha^{a}, \beta\right\rangle_{c}=a\langle\alpha, \beta\rangle_{c}, \\
\left\langle\alpha, \beta_{1}+_{F_{c}} \beta_{2}\right\rangle_{c} & =\left\langle\alpha, \beta_{1}\right\rangle_{c}+\left\langle\alpha, \beta_{2}\right\rangle_{c} ; & & \left\langle\alpha,[a]_{c}(\beta)\right\rangle_{c}=a\langle\alpha, \beta\rangle_{c} .
\end{aligned}
$$

Proof. Linearity over the first argument follows from that of the logarithmic derivative and the function $\ell(\alpha)$. Linearity over the second argument follows from that of the functions $\ell_{c}$ and $\lambda_{c}$.

Proposition 3. The pairing $\langle\cdot, \cdot\rangle_{c}$ satisfies the following Steinberg relation:

$$
\left\langle\alpha, \underline{c}^{p^{n}-1} \alpha\right\rangle_{c}=0, \text { where } \alpha \in X \mathcal{O}[[X]] \backslash\{0\} .
$$

Proof. First we prove the congruence

$$
\alpha^{m p}-\alpha^{m \Delta} \equiv m p \alpha^{m \Delta} \bmod (m p)^{2}, \text { for } \alpha \in \mathcal{O}[[X]]^{*} .
$$


By the definition of $\ell$ we have $\ell(\alpha)=\log \left(\alpha^{p} / \alpha^{\Delta}\right) / p$, i.e., $m p \cdot \ell(\alpha)=\log \alpha^{m p-m \Delta}$, whence

$$
\begin{aligned}
\alpha^{m p}-\alpha^{m \Delta} & =\alpha^{m \Delta}\left(\alpha^{m p-m \Delta}-1\right)=\alpha^{m \Delta}\left(\exp \left(\log \alpha^{m p-m \Delta}\right)-1\right) \\
& =\alpha^{m \Delta}(\exp (m p \cdot \ell(\alpha))-1)=\alpha^{m \Delta}\left(m p \ell(\alpha)+\sum_{i \geq 2} \frac{(m p)^{i}}{i !} \ell(\alpha)^{i}\right) \\
& =\alpha^{m \Delta} m p \ell(\alpha)+\alpha^{m \Delta} \sum_{i \geq 2} \frac{(m p)^{i}}{i !} \ell(\alpha)^{i} .
\end{aligned}
$$

Note that if $i \geq 2$ and $p \geq 3$, then $(m p)^{i} / i ! \equiv 0 \bmod (m p)^{2}$. Therefore, (2) is true.

The identities in (1) imply

$$
\begin{gathered}
\psi_{m}=\frac{\alpha^{p m}-\alpha^{\Delta m}}{p m} \alpha^{-1} \mathrm{~d} \alpha-\ell(\alpha) \mathrm{d} \frac{\alpha^{\Delta m}}{p m}=\mathrm{d} \chi_{m}, \\
\text { where } \chi_{m}=\frac{\alpha^{p m}-\alpha^{\Delta m}}{(p m)^{2}}-\frac{\alpha^{\Delta m}}{p m} \ell(\alpha) .
\end{gathered}
$$

Moreover, the series $\chi_{m}$ has integral coefficients, in accordance with (2).

Any series $\beta \in \mathcal{O}[[x]]$ satisfies the congruence

$$
\beta^{p^{n} m} \equiv \beta^{p^{n-1} m \Delta} \bmod p^{n} .
$$

Indeed, $\beta^{p} \equiv \beta^{\Delta} \bmod p$, and easy induction shows that $\beta^{p^{n}} \equiv \beta^{p^{n-1} \Delta} \bmod p^{n}$, yielding (4).

Now we calculate the series $\Phi\left(\alpha, \underline{c}^{p^{n}-1} \alpha\right)$. Directly from the definition, we deduce the relation

$$
\Phi\left(\alpha, \underline{c}^{p^{n}-1} \alpha\right)=\ell_{c}\left(\underline{c}^{p^{n}-1} \alpha\right) \alpha^{-1} \mathrm{~d} \alpha-\ell(\alpha) \underline{c}^{-1} \mathrm{~d} \frac{\triangle}{p}\left(\underline{c} \lambda\left(\underline{c}^{p^{n}-1} \alpha\right)\right) .
$$

Consider the first summand and apply the definition of $\ell_{c}$ to it:

$$
\begin{aligned}
\ell_{c}\left(\underline{c}^{p^{n}-1} \alpha\right) \alpha^{-1} \mathrm{~d} \alpha= & \underline{c}^{-1}\left(1-\frac{\Delta}{p}\right) \log \left(1+\underline{c}^{p^{n}} \alpha\right) \alpha^{-1} \mathrm{~d} \alpha \\
= & \underline{c}^{-1} \sum_{p \nmid m}(-1)^{m-1} \frac{\underline{c}^{p^{n} m} \alpha^{m}}{m} \alpha^{-1} \mathrm{~d} \alpha \\
& \quad+\underline{c}^{-1} \sum_{m \geq 1}(-1)^{m-1} \frac{\underline{c}^{p^{n+1} m} \alpha^{p m}-\underline{c}^{p^{n} \Delta m} \alpha^{\Delta m}}{p m} \alpha^{-1} \mathrm{~d} \alpha .
\end{aligned}
$$

It is easily seen that if $p \nmid m$, then

$$
\frac{\underline{c}^{p^{n} m} \alpha^{m}}{m} \alpha^{-1} \mathrm{~d} \alpha \equiv \mathrm{d}\left(\frac{\underline{c}^{p^{n} m} \alpha^{m}}{m^{2}}\right) \bmod p^{n},
$$

so that the first summand in (6) takes the form

$$
\underline{c}^{-1} \sum_{p \nmid m}(-1)^{m-1} \frac{c^{p^{n} m} \alpha^{m}}{m} \alpha^{-1} \mathrm{~d} \alpha \equiv \underline{c}^{-1} \mathrm{~d} \eta \bmod p^{n}, \quad \text { where } \eta=\sum_{p \nmid m}(-1)^{m-1} \frac{c^{p^{n} m} \alpha^{m}}{m^{2}} .
$$

Here the series $\eta$ has integral coefficients. Finally, substituting this in (6) and taking (4) into account, we get

$$
\ell_{c}\left(\underline{c}^{p^{n}-1} \alpha\right) \alpha^{-1} \mathrm{~d} \alpha=\underline{c}^{-1} \mathrm{~d} \eta+\underline{c}^{-1} \sum_{m \geq 1}(-1)^{m-1} \underline{c}^{p^{n+1} m} \frac{\alpha^{p m}-\alpha^{\Delta m}}{p m} \alpha^{-1} \mathrm{~d} \alpha .
$$


Now we consider the second summand in (5):

$$
\ell(\alpha) \underline{c}^{-1} \mathrm{~d} \frac{\triangle}{p}\left(\underline{c} \lambda\left(\underline{c}^{p^{n}-1} \alpha\right)\right)=\ell(\alpha) \underline{c}^{-1} \mathrm{~d} \sum_{m \geq 1}(-1)^{m-1} \frac{\underline{c}^{p^{n} \Delta m} \alpha^{\Delta m}}{p m} .
$$

We use congruence (4) to get

$$
\begin{aligned}
\ell(\alpha) \underline{c}^{-1} \mathrm{~d} \frac{\Delta}{p}\left(\underline{c} \lambda\left(\underline{c}^{p^{n}-1} \alpha\right)\right) & \equiv \ell(\alpha) \underline{c}^{-1} \mathrm{~d} \sum_{m \geq 1}(-1)^{m-1} \frac{\underline{c}^{p^{n+1}} m}{\alpha^{\Delta m}} \\
& \equiv \ell(\alpha) \underline{c}^{-1} \sum_{m \geq 1}(-1)^{m-1} \underline{c}^{p^{n+1} m} \mathrm{~d} \frac{\alpha^{\Delta m}}{p m} \bmod p^{n} .
\end{aligned}
$$

Hence, recalling (7), we see that

$$
\Phi\left(\alpha, \underline{c}^{p^{n}-1} \alpha\right) \equiv \underline{c}^{-1}\left(\mathrm{~d} \eta+\sum_{m \geq 1}(-1)^{m-1} \underline{c}^{p^{n+1} m}\left(\frac{\alpha^{p m}-\alpha^{\Delta m}}{p m} \alpha^{-1} \mathrm{~d} \alpha-\ell(\alpha) \mathrm{d} \frac{\alpha^{\Delta m}}{p m}\right)\right) .
$$

The definition of the function $\chi_{m}$ (see (3) ) implies

$$
\Phi\left(\alpha, \underline{c}^{p^{n}-1} \alpha\right) \equiv \underline{c}^{-1}\left(\mathrm{~d} \eta+\sum_{m \geq 1}(-1)^{m-1} \underline{c}^{p^{n+1} m} \mathrm{~d} \chi_{m}\right) \bmod p^{n},
$$

and the series $\varphi$ and $\chi_{m}$ have integral coefficients. Thus,

$$
\left\langle\alpha, \underline{c}^{p^{n}-1} \alpha\right\rangle_{c}=\operatorname{res}_{X} \Phi\left(\alpha, \underline{c}^{p^{n}-1} \alpha\right) / s_{c} \equiv \operatorname{res}_{X} \mathrm{~d}\left(\eta+\sum_{m \geq 1} \underline{c}^{p^{n+1} m} \chi_{m}\right) / s \equiv 0 \bmod p^{n},
$$

because $s_{c}=\underline{c}^{-1} s$ and $\mathrm{d}(1 / s) \equiv 0 \bmod p^{n}$. This proves the Steinberg relation is question.

\section{§4. Primary Elements}

In the multiplicative case, the $p^{n}$-primary elements in the field $K$ were constructed and investigated in [1. Recall that an element $\omega \in K^{*}$ is said to be $p^{n}$-primary if the extension $K\left(\sqrt[p^{n}]{\omega}\right) / K$ is unramified. Two types of primary elements were defined in [1].

The first type will be called the Hasse type; we construct it in the following way. Let $a \in \mathcal{O}$ and $A \in \mathcal{O}_{\widetilde{T}}$ be elements such that $A^{\Delta}-A=a$. Then the element $H(a)=$ $\left.E\left(p^{n} A \ell(\underline{\zeta})\right)\right|_{X=\pi}$ is $p^{n}$-primary, and for the Hilbert symbol $(\cdot, \cdot): K^{*} \times K^{*} \rightarrow\langle\zeta\rangle$ in the field $K$ we have $(\pi, H(a))=\zeta^{\operatorname{tr} a}$ (see [1, §4, Lemma 8]).

The second type of $p^{n}$-primary elements, as constructed in [1], has the form $\omega(a)=$ $\left.E\left(a\left(\underline{\zeta}^{p^{n}}-1\right)\right)\right|_{X=\pi}$; it is related to $H(a)$ by $\omega(a) \equiv H(a) \bmod \left(K^{*}\right)^{p^{n}}$ (see [1, $\S 4$, item 3]). The main difference between these types is that in the construction of the second only elements of initial field $K$ are used.

Denote $H^{\prime}(a)=\sqrt[p^{n}]{H(a)}, \omega^{\prime}(a)=\sqrt[p^{n}]{\omega(a)}$. Then $H^{\prime}(a)$ and $\omega^{\prime}(a)$ lie in the finite unramified extension of the field $T(\zeta)$.

Now we define primary elements in the formal module $F_{c}(\mathfrak{M})$. An element $\omega_{c} \in F_{c}(\mathfrak{M})$ is said to be $p^{n}$-primary if the extension $K\left(\frac{1}{\left[p^{n}\right]_{c}} \omega_{c}\right) / K$ is unramified. Define

$$
\begin{aligned}
& H_{c}(a)=c^{-1}(H(a)-1)=\left.E_{c}\left(p^{n} A \ell_{c}(\xi)\right)\right|_{X=\pi} ; \\
& \omega_{c}(a)=c^{-1}(\omega(a)-1)=\left.E_{c}\left(a s_{c}(X)\right)\right|_{X=\pi} .
\end{aligned}
$$

It is clear that $H_{c}(a), \omega_{c}(a) \in T(\zeta, c)$, and the elements $H_{c}^{\prime}(a)=\frac{1}{\left[p^{n}\right]_{c}} H_{c}(a), \omega_{c}^{\prime}(a)=$ $\frac{1}{\left[p^{n}\right]_{c}} \omega_{c}(a)$ lie in the unramified extension of the field $T(\zeta, c)=T(\xi, c)$.

Theorem 1. $H_{c}^{\prime}(a)^{\varphi}=H_{c}^{\prime}(a)+F_{c}\left[a_{\varphi}\right](\xi)$, where $A^{\varphi}-A=a_{\varphi} \in \mathbb{Z}_{p}$. 
Proof. Since a similar statement is true for the multiplicative case (see [1, $\S 4$, proof of Lemma 8]), and $c^{\varphi}=c$, we have

$$
\begin{aligned}
H_{c}^{\prime}(a)^{\varphi} & =c^{-1}\left(H^{\prime}(a)^{\varphi}-1\right)=c^{-1}\left(H^{\prime}(a) \zeta^{a_{\varphi}}-1\right) \\
& =c^{-1}\left(\left.E(A \ell(\underline{\zeta}))\right|_{X=\pi} \cdot \zeta^{a_{\varphi}}-1\right) \\
& =c^{-1}\left(\left(1+\left.\underline{c} E_{c}\left(A \underline{c}^{-1} \ell(\underline{\zeta})\right)\right|_{X=\pi}\right) \cdot \zeta^{a_{\varphi}}-1\right) \\
& =c^{-1}\left(\left(1+\left.\underline{c} E_{c}\left(A \ell_{c}(\underline{\xi})\right)\right|_{X=\pi}\right) \cdot \zeta^{a_{\varphi}}-1\right) \\
& =c^{-1}\left(\left(1+c H_{c}^{\prime}(a)\right) \cdot \zeta^{a_{\varphi}}-1\right)=H_{c}^{\prime}(a)+{ }_{F_{c}}\left[a_{\varphi}\right](\xi) .
\end{aligned}
$$

Remark 1. In our case $\varphi=\triangle^{f}$ with $f=\left(T: \mathbb{Q}_{p}\right)$, whence $A^{\varphi}-A=\operatorname{tr} a \Rightarrow a_{\varphi}=\operatorname{tr} a$.

Corollary. $\left(\pi, H_{c}(a)\right)_{c}=[\operatorname{tr} a]_{c}(\xi)$.

Proof. $H_{c}(a) \in T(\zeta, c), H_{c}^{\prime}(a) \in \widetilde{T}(\zeta, c)$. By the definition of the Hilbert symbol, we get

$$
\left(\pi, H_{c}(a)\right)_{c}=H_{c}^{\prime}(a)^{\varphi}-F_{c} H_{c}^{\prime}(a)=[\operatorname{tr} a]_{c}(\xi) .
$$

Proposition 4. $\omega_{c}(a) \equiv H_{c}(a) \bmod \left[p^{n}\right]_{c}\left(F_{c}(\mathfrak{M})\right)$.

Proof. A similar proposition for the multiplicative case (see [1, §4] or [3, Chapter V, item 4]) gives $\omega(a)=H(a) \cdot \varepsilon^{p^{n}}$, where $\varepsilon$ is some unit in $\mathcal{O}_{K}$ such that $\varepsilon \equiv 1 \bmod \pi$. Thus,

$$
\omega(a)=H(a) \cdot \varepsilon^{p^{n}}=\left(1+c H_{c}(a)\right) \cdot\left(1+c\left[p^{n}\right]_{c}\left(c^{-1}(\varepsilon-1)\right)\right) .
$$

Now, since $\omega_{c}(a)=c^{-1}(\omega(a)-1)$, we get

$$
\begin{aligned}
\omega_{c}(a) & =c^{-1}\left(\left(1+c H_{c}(a)\right) \cdot\left(1+c\left[p^{n}\right]_{c}\left(c^{-1}(\varepsilon-1)\right)\right)-1\right) \\
& =H_{c}(a)+{ }_{F_{c}}\left[p^{n}\right]_{c}\left(c^{-1}(\varepsilon-1)\right) .
\end{aligned}
$$

Proposition 5. Let $\zeta-1=\gamma_{e_{0}} \pi^{e_{0}}+\ldots$, where $\gamma_{e_{0}} \in \mathcal{O}$. Then

$$
\omega_{c}(a) \equiv H_{c}(a) \equiv c^{-1} a^{p^{n}} \gamma_{e_{0}}^{p^{n}} \pi^{p e_{1}} \bmod \left(\pi^{p e_{1}+1},\left[p^{n}\right]_{c}\left(F_{c}(\mathfrak{M})\right)\right) .
$$

Proof. It is known that

$$
H(a)=\left.E\left(p^{n} A \ell(\underline{\zeta})\right)\right|_{X=\pi} \equiv 1+a^{p^{n-1}} \gamma_{e_{0}}^{p^{n}} \pi^{p e_{1}} \bmod \pi^{p e_{1}+1}
$$

(see [2, p. 121, (14)]). Now the definition of $H_{c}(a)$ directly implies the formula

$$
H_{c}(a)=c^{-1}(H(a)-1) \equiv c^{-1} a^{p^{n-1}} \gamma_{e_{0}}^{p^{n}} \pi^{p e_{1}} \bmod \pi^{p e_{1}+1} .
$$

The final result follows from Proposition 4 .

Proposition 6. The primary element $\omega_{c}(a) \bmod \left[p^{n}\right]_{c}\left(F_{c}(\mathfrak{M})\right)$ does not depend on the choice of the prime element $\pi$ and the form of the series expansion for the root $\xi$ of the kernel $\left[p^{n}\right]_{c}(x)$.

Proof. The proof is much similar to that in the multiplicative case (see 3, Chapter V, item 4]).

\section{$\S 5$. Main Lemma}

First, we prove some preliminary assertions.

Lemma 1. For $\psi \in X \mathcal{O}[[X]]$, the following congruence holds true:

$$
\left(\frac{\triangle}{p} \log (1+\underline{c} u \psi)\right) / s \equiv\left(\frac{\triangle}{p} \log (1+p \underline{c} \psi)\right) / s \bmod \left(p^{n}, \operatorname{deg} 1\right) .
$$


Proof. For series $u$ and $s$, we have

$$
\frac{u^{r \Delta}}{r p} / s \equiv \frac{p^{r-1}}{r} / s \bmod \left(p^{n}, \operatorname{deg} 0\right), \quad \text { where } r \geq 1
$$

(see [4, proof of Lemma 4]). Multiplying both sides of this congruence by $(-1)^{r-1}(\underline{c} \psi)^{r \Delta}$ and summing over $r$, we arrive at (9).

Lemma 2. For $\psi \in X \mathcal{O}[[X]]$, the following congruence holds true:

$$
\log (1+\underline{c} u \psi) / s \equiv \frac{1}{p} \log (1+p \underline{c} \psi) / s_{n-1} \bmod \operatorname{deg} 1 .
$$

Proof. We use the congruence $u^{r} / s \equiv p^{r-1} / s_{n-1} \bmod \operatorname{deg} 0$, where $r \geq 1$ (see 4, proof of Lemma 3]). Multiplying by $(-1)^{r} \frac{(\underline{c} \psi)^{r}}{r}$ and summing over $r$, we complete the proof.

\section{Corollary.}

$$
\triangle(\log (1+\underline{c} u \psi) / s) \equiv\left(\frac{\Delta}{p} \log (1+p \underline{c} \psi)\right) / s \bmod \left(p^{n}, \operatorname{deg} 1\right) .
$$

Proof. It suffices to apply the operator $\Delta$ to both sides of (10) and to recall that $1 / s_{n-1}^{\Delta} \equiv$ $1 / s^{\Delta} \bmod p^{n}($ see [3, Chapter VI, item 3]).

Lemma 3. For $\psi \in X \mathcal{O}[[X]]$, the following congruence holds true:

$$
\frac{\triangle}{p} \log (1+\underline{c} u \psi) \mathrm{d}(1 / s) \equiv 0 \bmod \left(p^{n}, \operatorname{deg} 0\right)
$$

Proof. Note that $d(1 / s) \equiv 0 \bmod p^{n}$ (see [3, Chapter VI, item 3]). Since the series $\left(1-\frac{\Delta}{p}\right) \log (1+\underline{c} u \psi)$ has integral coefficients, we have

$$
\left(1-\frac{\triangle}{p}\right) \log (1+\underline{c} u \psi) \mathrm{d}(1 / s) \equiv 0 \bmod p^{n} .
$$

Now the claim is equivalent to the following congruence:

$$
\log (1+\underline{c} u \psi) \mathrm{d}(1 / s) \equiv 0 \bmod \left(p^{n}, \operatorname{deg} 0\right) .
$$

The definition of the series $u$ (see [1, §3]) shows that

$$
u \in p O[[X]]+X^{e} O[[X]] \Rightarrow \underline{c} u \psi=p a+X^{e} b, \text { where } a, b \in \mathcal{O}[[X]] .
$$

Plugging this in the series for $\log (1+X)$, we get

$$
\log (1+\underline{c} u \psi)=p a_{-1}+a_{0} X^{e}+a_{1} \frac{X^{p e}}{p}+a_{2} \frac{X^{p^{2} e}}{p^{2}}+a_{3} \frac{X^{p^{3} e}}{p^{3}}+\ldots,
$$

where $a_{i} \in \mathcal{O}[[X]]$. From the definition of $s$ and $\underline{\zeta}$ and the properties of $\zeta$ (see [2, p. 121]) it follows that

$$
\underline{\zeta} \in 1+p \mathcal{O}[[X]]+X^{e_{0}} \mathcal{O}[[X]] \Rightarrow s=p X^{p^{n} e_{0}-e} \varepsilon_{1}(X)+X^{p^{n} e_{0}} \varepsilon_{2}(X),
$$

where $\varepsilon_{1}, \varepsilon_{2} \in \mathcal{O}\{\{X\}\}^{*}$. This identity and the congruence $\mathrm{d} s \equiv 0 \bmod p^{n}$ (see [3. Chapter VI, item 3]) imply that

$$
\mathrm{d}(1 / s)=-\mathrm{d} s / s^{2}=p^{n}\left(b_{0} X^{-2 p^{n} e_{0}}+b_{1} X^{-2 p^{n} e_{0}-e} p+b_{2} X^{-2 p^{n} e_{0}-2 e} p^{2}+\ldots\right),
$$

where $b_{i} \in \mathcal{O}[[X]]$. The expansions (14) and (15) with the inequality $p^{m} e \geq-2 p^{n} e_{0}+m e$ for $m \geq 1$ prove the desired congruence (13).

Lemma 4 (main lemma). For $\alpha \in \mathcal{H}_{\mathfrak{m}}$ and $\psi \in X \mathcal{O}[[X]]$, the following congruence holds true:

$$
\langle\alpha, u \psi\rangle_{c} \equiv(1-\triangle) d_{0} \bmod p^{n}, \text { where } d_{0} \in \mathcal{O} \text {. }
$$


Proof. By the definition of the pairing $\langle\cdot, \cdot\rangle_{c}$, we have

$$
\langle\alpha, u \psi\rangle_{c}=\operatorname{res}_{X}\left(\ell_{c}(u \psi) \alpha^{-1} \mathrm{~d} \alpha\right) / s_{c}-\left(\ell(\alpha) \mathrm{d} \frac{\Delta}{p} \log (1+\underline{c} u \psi)\right) / s .
$$

Consider the second summand. Since $\ell(\alpha) \in \mathcal{O}[[X]]$, the obvious relation

$$
\operatorname{res}_{X} \mathrm{~d}\left(\left(\ell(\alpha) \frac{\triangle}{p} \log (1+\underline{c} u \psi)\right) / s\right)=0
$$

and congruence (12) imply

$$
-\operatorname{res}_{X}\left(\ell(\alpha) \mathrm{d} \frac{\Delta}{p} \log (1+\underline{c} u \psi)\right) / s \equiv \operatorname{res}_{X} \mathrm{~d} \ell(\alpha)\left(\frac{\triangle}{p} \log (1+\underline{c} u \psi)\right) / s .
$$

Directly from the definition of $\ell$ we see that

$$
\mathrm{d} \ell(\alpha)=\alpha^{-1} \mathrm{~d} \alpha-X^{-1}\left(X \alpha^{-1} \mathrm{~d} \alpha\right)^{\Delta} .
$$

Substituting the resulting representation of the second summand in (16), we obtain

$$
\begin{aligned}
& \langle\alpha, u \psi\rangle_{c} \equiv \operatorname{res}_{X}\left(\alpha^{-1} \mathrm{~d} \alpha\right)\left(\ell_{c}(u \psi) / s_{c}+\left(\frac{\Delta}{p} \log (1+\underline{c} u \psi)\right) / s\right) \\
& -X^{-1}\left(X \alpha^{-1} \mathrm{~d} \alpha\right)^{\Delta}\left(\frac{\triangle}{p} \log (1+\underline{c} u \psi)\right) / s \\
& \equiv \operatorname{res}_{X}\left(\alpha^{-1} \mathrm{~d} \alpha\right)\left(\ell(1+\underline{c} u \psi)+\frac{\Delta}{p} \log (1+\underline{c} u \psi)\right) / s \\
& -X^{-1}\left(X \alpha^{-1} \mathrm{~d} \alpha\right)^{\Delta}\left(\frac{\triangle}{p} \log (1+\underline{c} u \psi)\right) / s \\
& \equiv \text { [using the definition of } \ell \text { and formulas (11) and (9)] } \\
& \equiv \operatorname{res}_{X}\left(\alpha^{-1} \mathrm{~d} \alpha\right) \log (1+\underline{c} u \psi) / s-X^{-1}\left(X \alpha^{-1} \mathrm{~d} \alpha(\log (1+\underline{c} u \psi)) / s\right)^{\Delta} \\
& \equiv \operatorname{res}_{X} X^{-1}(1-\Delta)\left(X \alpha^{-1} \mathrm{~d} \alpha(\log (1+\underline{c} u \psi)) / s\right) \equiv(1-\Delta) d_{0} \bmod p^{n} \text {, }
\end{aligned}
$$

where $d_{0} \in \mathcal{O}$ is the constant term of the series $X \alpha^{-1} \mathrm{~d} \alpha(\log (1+\underline{c} u \psi)) / s$.

\section{§6. Hensel generators of the formal module $F_{c}(\mathfrak{M})$}

Lemma 5. The Hilbert symbol $(\cdot, \cdot)_{c}$ satisfies the following Steinberg type relation:

$$
\left(\alpha, c^{p^{n}-1} \alpha\right)_{c}=0 \text { for any } \alpha \in \mathfrak{M} \backslash\{0\} .
$$

Proof. We need to check that $\alpha$ is a multiplicative norm in the extension $K(\beta) / K$, where $\beta$ is a solution of the equation $\left[p^{n}\right]_{c}(X)=c^{p^{n}-1} \alpha$. Note that

$$
\left[p^{n}\right]_{c}(X)=c^{-1}\left((1+c X)^{p^{n}}-1\right)=p^{n} X+c_{2} X^{2}+\ldots+c_{p^{n}-1} X^{p^{n}-1}+c^{p^{n}-1} X^{p^{n}},
$$

where $c_{i} \in p \mathcal{O}_{K}$; hence $\beta$ solves the equation

$$
X^{p^{n}}+c^{1-p^{n}} c_{p^{n}-1} X^{p^{n}-1}+\ldots+p^{n} c^{1-p^{n}} X-\alpha=0,
$$

and, therefore, $\alpha$ is a norm in the extension $K(\beta) / K$.

Proposition 7. The elements

$$
\varepsilon_{i, \theta}=\theta c^{p^{n}-1} \pi^{i}, \text { where } \theta \in \Re, p \nmid i, \quad \text { and } \omega_{c}(a) \text { with } a \in \mathcal{O}
$$

generate the formal $\mathbb{Z}_{p}$-module $F_{c}(\mathfrak{M})$. Moreover, the Hilbert symbol $(\cdot, \cdot)_{c}$ satisfies

$$
\left(\pi, \varepsilon_{i, \theta}\right)_{c}=0, \quad\left(\pi, \omega_{c}(a)\right)=[\operatorname{tr} a]_{c}(\xi) .
$$


Proof. The first part of the proposition is a simple generalization of the Hensel theorem

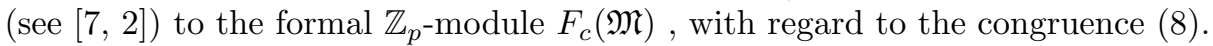

Now we check the relation $\left(\pi, \varepsilon_{i, \theta}\right)=0$. Indeed, Lemma 5 shows that

$$
0=\left(\theta \pi^{i}, c^{p^{n}-1}\left(\theta \pi^{i}\right)\right)_{c}=\left(\theta, c^{p^{n}-1} \theta \pi^{i}\right)_{c}+_{F_{c}}[i]_{c}\left(\pi, c^{p^{n}-1} \theta \pi^{i}\right)_{c}=[i]_{c}\left(\pi, c^{p^{n}-1} \theta \pi^{i}\right)_{c},
$$

because $\theta$ is a $p$-divisible element of the Teichmüller set $\mathfrak{R}$. Therefore, $\left(\pi, c^{p^{n}-1} \theta \pi^{i}\right)_{c}=0$ whenever $p \nmid i$. The second relation $\left(\pi, \omega_{c}(a)\right)_{c}=[\operatorname{tr} a]_{c}(\xi)$ is proved in the corollary to Theorem 1 and Proposition 4 .

Proposition 8. Let $\alpha \in \mathcal{H}_{\mathfrak{m}}, a \in \mathcal{O}$. Then

$$
\left\langle\alpha, E_{c}\left(a s_{c}(X)\right)\right\rangle_{c} \equiv \operatorname{deg} \alpha \cdot a \bmod p^{n} .
$$

Proof. First, we consider the case where $\alpha(X)=X$. By definition, we have

$$
\left\langle X, E_{c}\left(a s_{c}(X)\right)\right\rangle_{c}=\operatorname{res}_{X} \ell_{c}\left(E_{c}\left(a s_{c}(X)\right)\right) X^{-1} \mathrm{~d} X / s_{c}=\operatorname{res}_{X} a s_{c} X^{-1} / s_{c}=a .
$$

Since the pairing $\langle\cdot, \cdot\rangle_{c}$ is multiplicative in the first argument, it sufices to prove that for $\varepsilon(X) \in \mathcal{O}[[X]]^{*}$ we have

$$
\left\langle\varepsilon(X), E_{c}\left(a s_{c}(X)\right)\right\rangle_{c}=0
$$

Using the definition, we get

$$
\Phi\left(\varepsilon(X), E_{c}\left(a s_{c}(X)\right)\right) / s_{c}=a \varepsilon(X)^{-1} \mathrm{~d} \varepsilon(X)-\ell(\varepsilon(X)) \mathrm{d} \frac{\Delta}{p} \log \left(1+\underline{c} E_{c}\left(a s_{c}(X)\right)\right) / s .
$$

Trivially, we have $a \varepsilon(X)^{-1} \mathrm{~d} \varepsilon(X) \in \mathcal{O}[[X]]$, so that the residue of this element is zero. Consider the second summand:

$$
\begin{aligned}
& \quad(\varepsilon(X)) \mathrm{d} \frac{\Delta}{p} \log \left(1+\underline{c} E_{c}\left(a s_{c}(X)\right)\right) / s=\ell(\varepsilon) \mathrm{d} \frac{\Delta}{p} \log (E(a s)) / s \\
& \quad=\ell(\varepsilon) \cdot(1 / s) \mathrm{d} \sum_{m \geq 1} \frac{(a s)^{\Delta^{m}}}{p^{m}} \equiv \ell(\varepsilon) \cdot(1 / s) \cdot X^{-1}(X a \mathrm{~d} s)^{\Delta^{m}} \equiv 0 \bmod p^{n} .
\end{aligned}
$$

The last congruence is valid because $\mathrm{d} s \equiv 0 \bmod p^{n}$ and all other factors have integral coefficients. This proves formula (18) and the lemma.

\section{$\S 7$ PAIRING OVer the Formal Module $F_{c}(\mathfrak{M})$}

Now we introduce the pairing $\{\cdot, \cdot\}_{c}$ on numbers by using the pairing $\langle\cdot, \cdot\rangle_{c}$ on series.

Definition 1. Let

$$
\begin{aligned}
& \{\cdot, \cdot\}_{c}: K^{*} \times F_{c}(\mathfrak{M}) \rightarrow\langle\xi\rangle, \\
& \{\alpha, \beta\}_{c}=\left[\operatorname{tr}\langle\underline{\alpha}, \underline{\beta}\rangle_{c}\right]_{c}(\xi),
\end{aligned}
$$

where $\underline{\alpha} \in \mathcal{H}_{\mathfrak{m}}$ and $\underline{\beta} \in \mathcal{H}_{\mathrm{c}}$ are such that $\underline{\alpha}(\pi)=\alpha, \underline{\beta}(\pi)=\beta$.

In the following lemmas we check the consistency of this definition, i.e., the independence of the choice of the series $\underline{\alpha}$ and $\underline{\beta}$.

Lemma 6. Let series $\beta_{1}, \beta_{2} \in \mathcal{H}_{\mathrm{c}}$ be such that $\beta_{1}(\pi)=\beta_{2}(\pi)$. Then for any series $\alpha \in \mathcal{H}_{\mathfrak{m}}$ we have

$$
\left[\operatorname{tr}\left\langle\alpha, \beta_{1}\right\rangle_{c}\right]_{c}(\xi)=\left[\operatorname{tr}\left\langle\alpha, \beta_{2}\right\rangle_{c}\right]_{c}(\xi)
$$


Proof. Consider the series $\beta=\beta_{1}-F_{c} \beta_{2}$. By assumption, $\beta(\pi)=0$. Hence (see 1, Lemma 6$]$ ), $\beta=u \psi$ for some series $\psi \in X \mathcal{O}[[X]]$. The main lemma (Lemma 4) implies that

$$
\langle\alpha, \beta\rangle_{c}=0 \Rightarrow\left[\operatorname{tr}\langle\alpha, \beta\rangle_{c}\right]_{c}(\xi)=0 .
$$

Hence, by the linearity of the pairing $\langle\cdot, \cdot\rangle_{c}$, we get the claim.

Lemma 7 (change of variables). Let $g(Y) \in \mathcal{O}[[Y]]$ be such that $\operatorname{deg} g=1$, and let $g(\pi)=\pi^{\prime}$ be the uniformizing parameter of $\mathcal{O}_{K}$. Then for any $\alpha \in \mathcal{H}_{\mathfrak{m}}$ and $\beta \in \mathcal{H}_{\mathrm{c}}$ we have

$$
\langle\alpha(X), \beta(X)\rangle_{c, X, \pi}=\langle\alpha(g(Y)), \beta(g(Y))\rangle_{c, Y, \pi^{\prime}} .
$$

In the pairing on the right-hand side the series and residue are regarded with respect to the variable $Y$, and the corresponding series $s_{m}^{\prime}$ and $u^{\prime}$ are constructed with the help of expansions of $\xi, \zeta$, and $c$ in $\pi^{\prime}$-series (for the prime element $\pi^{\prime}$ ).

Proof. We denote by $\underset{\sim}{c}$ the $\pi^{\prime}$-series expansions of the element $c$.

First, we consider the case where $\alpha(X)=X$. By Lemma 6 and Proposition 7 , we may replace the series $\beta$ by the formal sum of the series $\theta \underline{c}^{p^{n}-1} X^{i}$ and $E_{c}\left(a s_{c}(X)\right)$. By the linearity of pairings, it suffices to check that

$$
\begin{aligned}
\left\langle X, \theta \underline{c}^{p^{n}-1} X^{i}\right\rangle_{c, X, \pi} & =\left\langle g(Y), \theta \underset{\sim}{\left.c^{p^{n}-1} g(Y)^{i}\right\rangle_{c, Y, \pi^{\prime}}, \text { where } p \nmid i,}\right. \\
\left\langle X, E_{c}\left(a s_{c}(X)\right)\right\rangle_{c, X, \pi} & =\left\langle g(Y), E_{c}\left(a s_{c}^{\prime}(Y)\right)\right\rangle_{c, Y, \pi^{\prime}} .
\end{aligned}
$$

The first congruence follows immediately from the Steinberg relation (Proposition 3), and the second from Proposition 8 .

Now we address the case of $\alpha(X)=\varepsilon(X) \in \mathcal{O}[[X]]^{*}$. It suffices to check only this case, by multipilicativity in the first argument. We need to prove that

$$
\langle\varepsilon(X), \beta(X)\rangle_{c, X, \pi}=\langle\varepsilon(g(Y)), \beta(g(Y))\rangle_{c, Y, \pi^{\prime}} .
$$

Denoting $f(X)=X \varepsilon(X), \pi^{\prime \prime}=f(\pi), Z=f(X)$, and $h(Y)=f(g(Y))$, we write

$$
\begin{aligned}
\langle\varepsilon(X), \beta(X)\rangle_{c, X, \pi} & =\langle f(X), \beta(X)\rangle_{c, X, \pi}-\langle X, \beta(X)\rangle_{c, X, \pi}, \\
\langle\varepsilon(g(Y)), \beta(g(Y))\rangle_{c, Y, \pi^{\prime}} & =\langle f(g(Y)), \beta(g(Y))\rangle_{c, Y, \pi^{\prime}}-\langle g(Y), \beta(g(Y))\rangle_{c, Y, \pi^{\prime}} .
\end{aligned}
$$

It suffices to prove that

$$
\langle f(X), \beta(X)\rangle_{c, X, \pi}=\langle f(g(Y)), \beta(g(Y))\rangle_{c, Y, \pi^{\prime}} .
$$

The case considered above shows that

$$
\begin{aligned}
\langle f(X), \beta(X)\rangle_{c, X, \pi} & =\left\langle Z, \beta\left(f^{-1}(Z)\right)\right\rangle_{c, Z, \pi^{\prime \prime}} \\
& =\left\langle h(Y), \beta\left(f^{-1}(h(Y))\right)\right\rangle_{c, Y, \pi^{\prime}}=\langle f(g(Y)), \beta(g(Y))\rangle_{c, Y, \pi^{\prime}} .
\end{aligned}
$$

The lemma is proved.

Lemma 8. Let series $\alpha_{1}, \alpha_{2} \in \mathcal{H}_{\mathfrak{m}}$ be such that $\alpha_{1}(\pi)=\alpha_{2}(\pi)$. Then for any series $\beta \in \mathcal{H}_{\mathrm{c}}$ we have

$$
\left[\operatorname{tr}\left\langle\alpha_{1}, \beta\right\rangle_{c}\right]_{c}(\xi)=\left[\operatorname{tr}\left\langle\alpha_{2}, \beta\right\rangle_{c}\right]_{c}(\xi) .
$$

Proof. By multiplicativity, it suffices to prove that

$$
\left\langle\alpha_{1} / \alpha_{2}, \beta\right\rangle_{c}=0 \text {. }
$$

Consider the series $g(X)=X \cdot\left(\alpha_{1}(X) / \alpha_{2}(X)\right)$; since $g(\pi)=\pi$, Lemmas [6] and [7 show that

$$
\langle X, \beta(X)\rangle_{c}=\langle g(X), \beta(g(X))\rangle_{c}=\langle g(X), \beta(X)\rangle_{c}=\left\langle X \cdot\left(\alpha_{1}(X) / \alpha_{2}(X)\right), \beta(X)\right\rangle_{c},
$$

which immediately implies the claim. 
We unite and reshape these results as follows.

Theorem 2. The pairing $\{\cdot, \cdot\}_{c}$ is well defined, i.e., it does not depend of the choice of a uniformizing element $\pi \in \mathcal{O}_{K}$ and the way of expansion of elements $\alpha \in K^{*}$ and $\beta \in$ $F_{c}(\mathfrak{M})$ in the $\pi^{\prime}$-series $\underline{\alpha} \in \mathcal{H}_{\mathfrak{m}}$ and $\beta \in \mathcal{H}_{\mathrm{c}}$. It is multiplicative in the first argument and linear in the second; moreover, it satisfies the Steinberg relation

$$
\left\{\alpha, c^{p^{n}-1} \alpha\right\}_{c}=0 \text {, where } \alpha \in F_{c}(\mathfrak{M}) \backslash\{0\} .
$$

\section{§8. HILBERT PAIRING}

Theorem 3. For any elements $\alpha \in K^{*}$ and $\beta \in F_{c}(\mathfrak{M})$, the values of the pairings $\{\cdot, \cdot\}_{c}$ and $(\cdot, \cdot)_{c}$ are equal:

$$
\{\alpha, \beta\}_{c}=(\alpha, \beta)_{c} .
$$

Proof. First, we consider case of prime $\alpha=\pi$. Expand $\beta$ in Hensel generators. By linearity of the pairings with respect to the second argument, it suffices to prove the theorem in the cases where $\beta=\varepsilon_{i, \theta}, \beta=\omega_{c}(a)$ (see Proposition 17). The Steinberg relations immediately imply (see the proof of Proposition 7) that

$$
\left\{\pi, \varepsilon_{i, \theta}\right\}_{c}=\left(\pi, \varepsilon_{i, \theta}\right)_{c}=0 .
$$

Propositions 7 and 8 also show that

$$
\left\{\pi, \omega_{c}(a)\right\}_{c}=\left(\pi, \omega_{c}(a)\right)_{c}=[\operatorname{tr} a](\xi) .
$$

The case of prime $\alpha$ is proved. Now, an arbitrary unit of the field $\varepsilon \in \mathcal{O}_{K}$ can be rewriten in the form $\varepsilon=\tau / \pi$, where $\tau$ and $\pi$ are prime elements. Then

$$
\{\varepsilon, \beta\}_{c}=\{\tau, \beta\}_{c}-F_{c}\{\pi, \beta\}_{c}=(\tau, \beta)_{c}-F_{c}(\pi, \beta)_{c}=(\varepsilon, \beta)_{c} .
$$

By multplicativity in the first argument, we get the desired claim for arbitrary $\alpha \in K^{*}$.

Thus, we have obtained an explicit formula for the symbol $(\cdot, \cdot)_{c}$.

\section{REFERENCES}

[1] S. V. Vostokov, An explicit form of the reciprocity law, Izv. Akad. Nauk SSSR Ser. Mat. 42 (1978), no. 6, 1288-1321; English transl., Math. USSR-Izv. 13 (1978), no. 3, 557-588. MR.522940 (80f:12014)

[2] I. R. Shafarevich, A general reciprocity law, Mat. Sb. 26 (1950), no. 1, 113-146. (Russian) MR0031944(11:230f)

[3] I. V. Fesenko and S. V. Vostokov, Local fields and their extensions, 2nd ed., Trans. Math. Monogr., vol. 121, Amer. Math. Soc., Providence, RI, 2002. MR1915966 (2003c:11150)

[4] S. V. Vostokov, The Hilbert symbol in a discretely valued field, Zap. Nauchn. Sem. Leningrad. Otdel. Mat. Inst. Steklov. (LOMI) 94 (1979), 50-69; English transl., J. Sov. Math. 19 (1982), no. 2, 1006-1019. MR571515 (81g:12019)

[5] S. V. Vostokov and E. V. Ferens-Sorotskiy, Hilbert pairing for the polynomial formal groups, Vestnik S.-Peterburg. Univ. Mat. 2010, vyp. 1, 23-27; English transl., Vestnik St. Petersburg Univ. Math. 43 (2010), no. 1, 18-22. MR2662405 (2011e:14085)

[6] S. V. Vostokov, V. V. Volkov, and G. K. Pak, The Hilbert symbol for polynomial formal groups, Zap. Nauchn. Sem. S.-Peterburg. Otdel. Mat. Inst. Steklov. (POMI) 400 (2012), 127-131; English transl., J. Math. Sci. (N.Y.) 192 (2013), no. 2, 196-199. MR3029567

[7] K. Hensel, Die multiplicative Dars ellung der algebraischen Zahlen fur den Bereich eines beliebigen Prin teil, J. Reine Angew. Math. 136 (1916).

[8] S. V. Vostokov, On I. R. Shafarevich's paper "A general reciprocity law", Mat. Sb. 400 (2013), no. 6, 3-22; English transl., Sb. Math. 204 (2013), no. 5-6, 781-800. MR3113452

[9] _ Normed pairing in formal modules, Izv. Akad. Nauk SSSR Ser. Mat. 43 (1979), no. 4, 765-794; English transl., Math. USSR-Izv. 15 (1980), no. 1, 25-51. MR548504 (81b:12017)

[10] _ Symbols on formal groups, Izv. Akad. Nauk SSSR Ser. Mat. 45 (1981), no. 5, 985-1014; English transl., Math. USSR-Izv. 19 (1982), no. 2, 261-284. MR637613 (83c:12016) 
[11] D. G. Benua and S. V. Vostokov, Norm pairing in formal groups and Galois representations, Algebra i Analiz 2 (1990), no. 6, 69-97; English transl., Leningrad Math. J. 2 (1991), no. 6, 12211249. MR 1092526 (92j:11141)

[12] V. A. Abrashkin, Explicit formulas for the Hilbert symbol of a formal group over Witt vectors, Izv. Ross. Akad. Nauk Ser. Mat. 61 (1997), no. 3, 3-56; English transl., Izv. Math. 61 (1997), no. 3, 463-515. MR $1478558(98 \mathrm{j}: 14060)$

[13] S. V. Vostokov and O. V. Demchenko, Explicit form on the Hilbert pairing for relative formal LubinTate groups, Zap. Nauchn. Sem. S.-Peterburg. Otdel. Mat. Inst. Steklov. (POMI) 227 (1995), 41-44; English transl., J. Math. Sci. (N.Y.) 89 (1998), no. 2, 1105-1107. MR1374555 (97b:11142)

[14] _ An explicit formula for the Hilbert pairing of formal Honda groups, Zap. Nauchn. Sem. S.-Peterburg. Otdel. Mat. Inst. Steklov. (POMI) 272 (2000), 86-128; English transl., J. Math. Sci. (N.Y.) 116 (2003), no. 1, 2926-2952. MR1811794 (2002k:11213)

Mathematics and Mechanics Department, St. Petersburg State University, Universitetskil Pr. 28, Petrodvorets, St. Petersburg 198504, Russia

E-mail address: sergei.vostokov@gmail.com

Mathematics and Mechanics Department, St. Petersburg State University, Universitetskiı Pr. 28, Petrodvorets, St. Petersburg 198504, Russia

E-mail address: vladvolkov239@gmail.com

Received 10/JAN/2014

Translated by V. VOLKOV 